\title{
THE UNIQUENESS OF POSITIVE SOLUTIONS OF PARABOLIC EQUATIONS OF DIVERGENCE FORM ON AN UNBOUNDED DOMAIN
}

\author{
MASAHARU NISHIO
}

\section{\$1. Introduction}

Let $R^{n+1}=R^{n} \times R$ be the $(n+1)$-dimensional Euclidean space $(n \geq 1)$. For $X \in R^{n+1}$, we write $X=(x, t)$ with $x \in R^{n}$ and $t \in R$. We consider parabolic operators of the following form:

$$
L=\frac{\partial}{\partial t}-\sum_{i, j=1}^{n} \frac{\partial}{\partial x_{i}} a_{i j}(x, t) \frac{\partial}{\partial x_{j}}
$$

where the coefficients $a_{\imath \jmath}$ are measurable functions with $a_{\imath \jmath}=a_{\imath_{\imath}}$ and satisfy

$$
M^{-1}|\xi|^{2} \leq \sum_{i, j=1}^{n} a_{i j}(x, t) \xi_{i} \xi_{j}, a_{i j}(x, t) \leq M
$$

with some positive constant $M$, for every $\xi=\left(\xi_{1}, \ldots, \xi_{n}\right) \in R^{n}$ and almost all $(x, t) \in R^{n+1}$

For an unbounded domain $\Omega$ in $R^{n+1}$, we put

$$
H_{0}(\Omega, L)=\left\{u \geq 0 ; L u=0 \text { on } \Omega, u=0 \text { on } \partial_{p} \Omega\right\},
$$

where $\partial_{p} \Omega$ denotes the parabolic boundary of $\Omega$.

In this paper, we assume that for every $\tau \in R, D_{\tau}=\left\{x \in R^{n} ;(x, \tau) \in \Omega\right\}$ is a bounded Lipschitz domain. Then $H_{0}(\Omega)$ coincides with $H_{0}\left(\Omega \cap R^{n} \times(-\infty\right.$, a)) for every $a \in R$. For a bounded Lipschitz domain $D$ in $R^{n}$ and a continuous function $\varphi>0$ on $(-\infty, a)$, we put

$$
\Omega(D, \varphi)=\left\{(x, t) \in R^{n+1} ; t<a, \varphi(t)^{-1} x \in D\right\} .
$$

By using a special form of the boundary Harnack principle for $\Omega(D, \varphi)$, we shall show the following

Received October 24, 1991. 
THEOREM 1. Let $D$ be a bounded Lipschitz domain in $R^{n}$ and $\varphi>0$ a $1 / 2$-Hölder continuous function on $(-\infty, a)$ for some $a \in(-\infty, \infty]$. If $\liminf |\tau|^{-1 / 2} \varphi(\tau)<\infty$, then there exists $u \neq 0$ such that

$$
H_{0}(\Omega(D, \varphi), L)=\{c u ; c \geq 0\} .
$$

\section{§2. Some estimates of $L$-parabolic measures}

For a domain $\Omega$ in $R^{n+1}$ and a point $(x, t)$ in $\Omega$, we denote by $\omega_{\Omega}^{(x, t)}$ the $L$-parabolic measure at $(x, t)$ with respect to $\Omega$.

First we recall the Aronson estimate of the fundamental solution of $L$. For an $M>0$, we denote by $\mathscr{L}(M)$ the class of the parabolic operators of the form (1) satisfying (2).

Lemma 1 (see [1]). Let $\Gamma(x, t ; y, s)$ be the fundamental solution of $L \in \mathscr{L}(M)$. Then there exist positive constants $C_{1}, C_{2}, \gamma_{1}, \gamma_{2}$ depending only on $M, n$ such that for all $(x, t),(y, s) \in R^{n+1}$,

$$
C_{1} g_{\gamma_{1}}(x, t ; y, s) \leq \Gamma(x, t ; y, s) \leq C_{2} g_{\gamma_{2}}(x, t ; y, s),
$$

where $g_{r}$ is the fundamental solution of $\partial / \partial t-\gamma \Delta$.

We shall use parabolic dilations. For $\alpha>0$, we denote by $\tau_{\alpha}$ the parabolic dilation defined by $\tau_{\alpha}(x, t)=\left(\alpha x, \alpha^{2} t\right)$. We note that $\mathscr{L}(M)$ is invariant for every parabolic dilation, that is, for any $L \in \mathscr{L}(M)$ and $\alpha>0, L_{\alpha} \in \mathscr{L}(M)$, where $L_{\alpha}\left(u^{\circ} \tau_{\alpha}\right)=L u$.

For a closed ball $B$ in $R^{n}$, we put

$$
T(B)=\left\{(x, t) ; t<0,(-t)^{-1 / 2} x \in B\right\},
$$

and for $r>0$ and a starlike open neighborhood $V$ of 0 in $R^{n}$, we put

$$
V_{r}=\left\{(x, t) ; r^{-1} x \in V,|t|<r^{2}\right\} \text {. }
$$

Lemma 2. Let $V$ be a starlike open neighborhood of 0 in $R^{n}$ and $B$ a closed ball contained in $V$. For $0<s<1$, there exists $\nu>0$ such that for any $L \in \mathscr{L}(M)$ and $X \in V_{s}$,

$$
\omega_{V_{1}}^{X}\left(\partial V_{1} \cap T(B)>\nu\right.
$$

Proof. Take a closed ball $B_{1}$ contained in the interior of $B$. Put 


$$
v(x, t)=\int_{R^{n} \backslash B_{1}} \Gamma(x, t ; y,-1) d y
$$

and

$$
w(x, t)=\omega_{V_{1}}^{(x, t)}\left(\partial V_{1} \cap T(B)\right) .
$$

By Lemma 1,

$$
v(x, t) \geq C_{1} \int_{R^{n} \backslash B_{1}} g_{\gamma_{1}}(x, t ; y,-1) d y,
$$

so that by the maximum principle there exists a constant $K>0$ such that

$$
1-w \leq K v \text { on } V_{1} \text {. }
$$

By Lemma 1 , we can choose $(\xi, \tau) \in V_{1}$ with $-1<\tau<-s^{2}$ such that

$$
v(\xi, \tau)<\frac{1}{2 K}
$$

By the Harnack inequality (see [4], p. 102), for any $(x, t) \in V_{s}$,

$$
w(x, t) \geq C w(\xi, \tau)>\frac{C}{2},
$$

which shows Lemma 2.

Remark 1. By using parabolic dilations, Lemma 2 implies that for $r>0$ and for $0<s<1$,

$$
\omega_{V_{r}}^{X}\left(\partial V_{r} \cap T(B)\right)>\nu \text { for } \quad X \in V_{s r}
$$

where $\nu$ is the constant in Lemma 2.

The above lemma gives the following

Lemma 3. Let $V$ be a starlike open neighborhood of 0 in $R^{n}$ and $B$ a closed ball contained in $V$. For any $\varepsilon>0$, there exists $s>0$ such that for any $L \in \mathscr{L}(M)$ and $X \in V_{s r} \backslash T(B)$,

$$
\omega_{V_{r} \backslash T(B)}^{X}\left(\partial V_{r} \backslash T(B)\right)<\varepsilon .
$$

This shows that 0 is a regular point in $V_{r} \backslash T(B)$ with respect to the Dirichlet problem. 
Proof. By using parabolic dilations, we may assume that $r=1$. For $L \in \mathscr{L}(M)$, we put

$$
u_{L}(x, t)=\omega_{V_{1} \backslash T(B)}^{X}\left(\partial V_{1} \backslash T(B)\right) .
$$

For $0<s<1$ and $(x, t) \in V_{s}$, we have

$$
u_{L}(x, t) \leq \omega_{V_{1}}^{(x, t)}\left(\partial V_{1} \backslash T(B)\right) \leq 1-\nu,
$$

where $\nu$ is the constant in Lemma 2. Since $u_{L}{ }^{\circ} \tau_{s}(x, t)=u_{L}\left(s x, s^{2} t\right)$ is a solution of $L_{s} u=0$, by the maximum principle,

$$
u_{L}{ }^{\circ} \tau_{s} \leq(1-\nu) u_{L_{s}} \text { on } \quad V_{1} \backslash T(B),
$$

and inductively we have for every integer $k>0$,

$$
u_{L^{\circ}} \tau_{s^{k}} \leq(1-\nu)^{k} u_{L_{s^{k}}} \text { on } \quad V_{1} \backslash T(B),
$$

which implies

$$
u_{L} \leq(1-\nu)^{k} \quad \text { on } \quad V_{s^{k}} \backslash T(B) .
$$

This shows Lemma 3.

\section{§3. The existence of positive solutions}

A domain $\Omega$ in $R^{n+1}$ is said to be spatially bounded if for every $\tau \in R, D_{\tau}=$ $\left\{x \in R^{n} ;(x, \tau) \in \Omega\right\}$ is bounded. A domain $\Omega$ in $R^{n+1}$ is called a $(1,1 / 2)$ Lipschitz domain with the Lipschitz constant $m$ if for every boundary point $(y, s) \in \partial \Omega$, there exist a coordinate system $\left(x_{1}, \ldots, x_{n}\right)$ of $R^{n}$, a function $f$ on $R^{n-1} \times R$ and a neighborhood $U$ of $(y, s)$ such that for every $x^{*}, \xi^{*} \in R^{n-1}$ and every $t, \tau \in R$,

$$
\left|f\left(x^{*}, t\right)-f\left(\xi^{*}, \tau\right)\right| \leq m\left(\left|x^{*}-\xi^{*}\right|+|t-\tau|^{1 / 2}\right)
$$

and

$$
\Omega \cap U=\left\{\left(x^{*}, x_{n}, t\right) \in U ; x_{n}>f\left(x^{*}, t\right)\right\} .
$$

Let $D$ be a bounded Lipschitz domain in $R^{n}, \tau \in R$ and $m>0$. A point $X \in R^{n+1}$ is called a proper inner point with respect to $(D, \tau, m)$ if $X \in \Omega$ for every (1, 1/2)-Lipschitz domain $\Omega$ with the Lipschitz constant $m$ satisfying $\left\{x \in R^{n}\right.$; $(x, \tau) \in \Omega\}=D$.

Hereafter we shall give a special form of the boundary Harnack principle, which is used to show the existence of a non-zero solution in $H_{0}(\Omega, L)$. 
Lemma 4. Let $\Omega$ be a spatially bounded $(1,1 / 2)$-Lipschitz domain in $R^{n+1}$ with the Lipschitz constant $m$. For $\tau \in R$, we put $D=D_{\tau}$. For $x_{0} \in R^{n}$ and $\tau_{0}>0$, we assume that $\left(x_{0}, \tau+\tau_{0}\right)$ is a proper inner point with respect to $(D, \tau, m)$. Then there exists a constant $C>0$ such that for any solution $u \geq 0$ of $L u=0$ on $\Omega^{(\tau)}=$ $\Omega \cap R^{n} \times(\tau, \infty)$ which vanishes continuously on $\partial \Omega \cap R^{n} \times[\tau, \infty)$,

$$
u(x, t) \leq C u\left(x_{0}, \tau+t_{0}\right) \text { for }(x, t) \in \Omega^{\left(\tau+\tau_{0}\right)},
$$

where $C$ depends only on $n, M, m, D, x_{0}$ and $\tau_{0}$.

Proof. Put $V=\left\{\left(x_{1}, \ldots, x_{n}\right) ;|x|<,3 m, j=1, \ldots, n\right\}$. For $r>0$ and $Y_{0} \in$ $R^{n+1}$, we set $V_{r}\left(Y_{0}\right)=\left\{Y_{0}\right\}+V_{r}$ (for the notation $V_{r}$, see the paragraph 2). If a solution $u \geq 0$ of $L u=0$ on $\Omega^{(\tau)}$ vanishes continuously on $\partial \Omega \cap R^{n} \times[\tau, \infty)$, then for any $(x, t) \in \Omega^{(\tau)}$

$$
u(x, t)=\int_{D \times\{\tau\}} u(y, \tau) d \omega_{\Omega^{(\tau)}}^{(x, t)}(y),
$$

and the parabolic measure $\omega_{\Omega^{(\tau)}}^{(x, t)}$ is absolutely continuous with respect to $\omega_{\Omega^{(\tau)}}^{\left(x_{0}, \tau+\tau_{0}\right)}$ on $D \times\{\tau\}$. Hence it suffices to show that

$$
\omega_{\Omega^{(\tau)}}^{(x, t)}\left(V_{r}\left(y_{0}, \tau\right)\right) \leq C \omega_{\Omega^{(\tau)}}^{\left(x_{0}, \tau+\tau_{0}\right)}\left(V_{r}\left(y_{0}, \tau\right)\right)
$$

for $(x, t) \in \Omega^{\left(\tau+\tau_{0}\right)}$ and sufficiently small $r>0$. As $\Omega$ is $(1,1 / 2)$-Lipschitz, there exist a finite family $\left(U_{k}\right)$ of open sets in $R^{n+1}$ with $\cup U_{k} \supset \partial D \times\{\tau\}$ such that $U_{k}$ associates with a coordinate system and a function satisfying (3). If $\left(y_{0}, \tau\right) \notin D \times\{\tau\} \backslash \cup U_{k}$, we put $A_{r}\left(y_{0}, \tau\right)=\left(y_{0}, \tau+2 r^{2}\right)$. Otherwise we choose another open set $U$ in $R^{n+1}$, an associated coordinate system in $R^{n+1}$ and a function $f$ satisfying (3). Put $A_{r}\left(y_{0}, \tau\right)=\left(y_{0}^{*}, y_{0 n}+3 m r, \tau+2 r^{2}\right)$, where $y_{0}=$ $\left(y_{0}^{*}, y_{0 n}\right) \in R^{n-1} \times R$, and

$$
v(x, t)=\omega_{\Omega^{(\tau)}}^{(x, t)}\left(V_{r}\left(y_{0}, \tau\right)\right)
$$

We shall show that there exists $C_{0}>0$ such that

$$
v(x, t)=C_{0} \omega_{\Omega^{(\tau)}}^{(x, t)}\left(A_{2^{k} r}\left(y_{0}, \tau\right)\right), \quad(x, t) \in \Omega^{(\tau)} \backslash V_{2^{k} r}\left(y_{0}, \tau\right)
$$

for every integer $k \geq 0$ with $2^{2 k+1} r^{2} \geq t_{0} / 2$. By Remark 1 and the Harnack inequality, we have for some $C_{1}>0$,

$$
v(x, t) \leq 1 \leq \frac{1}{\nu} v\left(A_{r / 2}\left(y_{0}, \tau\right)\right) \leq \frac{C_{1}}{\nu} v\left(A_{r}\left(y_{0}, \tau\right)\right) .
$$

Similarly 


$$
v\left(A_{r}\left(y_{0}, \tau\right)\right) \leq C_{1} v\left(A_{2 r}\left(y_{0}, \tau\right)\right)
$$

so that

$$
v(x, t) \leq \frac{C_{1}^{2}}{\nu} v\left(A_{2 r}\left(y_{0}, \tau\right)\right), \quad(x, t) \in \Omega^{(\tau)} \backslash V_{r}\left(y_{0}, \tau\right)
$$

By using Lemma 3 for $\varepsilon=1 / C_{1}$ and for $B=\left\{\left(x^{*}, x_{n}\right) \in R^{n} ;\left|x^{*}\right|^{2}+\left(x_{n}+\right.\right.$ $\left.2 m)^{2} \leq m^{2} /\left(1+m^{2}\right)\right\}$, there exists $0<s<1$ such that

$$
\omega_{V_{r}(Y) \backslash(\{Y\}+T(B))}^{X}(\{Y\}+T(B))<\frac{1}{C_{1}}, \quad X \in V_{s r}(Y) \backslash(\{Y\}+T(B))
$$

for every $Y \in R^{n+1}$. Hence for every $Y \in \partial \Omega^{(\tau)} \backslash V_{2 r}\left(y_{0}, \tau\right)$ and $(x, t) \in V_{s r}(Y)$,

$$
\begin{aligned}
v(x, t) & \leq \frac{C_{1}^{2}}{\nu} v\left(A_{2 r}\left(y_{0}, \tau\right)\right) \omega_{V_{r}(Y) \backslash(\{Y\}+T(B))}^{(x, t)}(\{Y\}+T(B)) \\
& \leq \frac{C_{1}}{\nu} v\left(A_{2 r}\left(Y_{0}, \tau\right)\right) .
\end{aligned}
$$

On the other hand, for every $(x, t) \in \partial V_{2 r}\left(y_{0}, \tau\right)$ which is not included in any $V_{s r}(Y)$ with $Y \in \partial \Omega^{(\tau)} \backslash V_{2 r}\left(y_{0}, \tau\right)$, the Harnack inequality gives

$$
v(x, t) \leq C_{2} v\left(A_{2 r}\left(y_{0}, \tau\right)\right)
$$

with some constant $C_{2}>0$. Therefore by the maximum principle, we have

$$
v(x, t) \leq C_{0} v\left(A_{2 r}\left(y_{0}, \tau\right)\right), \quad(x, t) \in \Omega^{(\tau)} \backslash V_{2 r}\left(y_{0}, \tau\right)
$$

for $C_{0}=\max \left(C_{1} / \nu, C_{2}\right)$, which shows (5) for $k=1$. Thus inductively we have (5) for every integer $k \geq 0$.

Furthermore we have

$$
v\left(A_{t_{0}^{1 / 2} / 2}\left(y_{0}, \tau\right)\right) \leq C_{3} v\left(x_{0}, \tau+t_{0}\right)
$$

by the Harnack inequality, where $C_{3}>0$ is a constant depending only on $n, M$, $m, D, x_{0}$ and $t_{0}$. Combining (5) and (6), we obtain (4), which shows Lemma 4.

This gives the following

Lemma 5. In the same situation as in Lemma 4, we have

$$
u(x, t) \leq C u\left(x_{0}, \tau+\tau_{0}\right) \omega_{\Omega^{\left(\tau+\tau_{0}\right)}}^{(x, t)}\left(D_{\tau+\tau_{0}} \times\left\{\tau+\tau_{0}\right\}\right)
$$

for every $(x, t) \in \Omega^{\left(\tau+\tau_{0}\right)}$, where $C>0$ is the constant in Lemma 4 . 
Using the above two lemmas, we obtain the Harnack inequality of the following form.

Proposition 1. Let $\Omega$ be a spatially bounded (1,1/2)-Lipschitz domain in $R^{n+1}, \tau \in R$ and $K$ a compact subset of $\Omega^{(\tau)}$. Then there exists a constant $C>0$ such that for every $L \in \mathscr{L}(M)$ and every solution $u \geq 0$ of $L u=0$ on $\Omega^{(\tau)}$ which vanishes continuously on $\partial \Omega \cap R^{\mathrm{n}} \times[\tau, \infty)$,

$$
\max _{K} u \leq C \min _{K} u .
$$

In [2], E.B. Fabes, N. Garofalo and S. Salsa show a similar Harnack inequality in the case $\Omega$ is a Lipschitz cylinder.

We shall prove the existence of non-zero $u \in H_{0}(\Omega, L)$ by using Lemma 5 and Proposition 1.

Proposition 2. Let $\Omega$ be a spatially bounded (1,1/2)-Lipschitz domain in $R^{n+1}$. Then there exists a non-zero positive solution $u$ of $L u=0$ on $\Omega$ such that $u$ vanishes continuously on $\partial \Omega$.

Proof. Let $Y_{0}=\left(y_{0}, s_{0}\right) \in \Omega$ be fixed. For $\tau<s_{0}$, we put

$$
u_{\tau}(x, t)=\frac{\omega_{\Omega^{(\tau)}}^{(x, t)}\left(D_{\tau} \times\{\tau\}\right)}{\omega_{\Omega^{(\tau)}}^{Y_{0}}\left(D_{\tau} \times\{\tau\}\right)} .
$$

Then $u_{\tau}\left(Y_{0}\right)=1$. Therefore by Proposition 1 , for every $t_{0}<s_{0}$, the sequence $\left\{u_{\tau}\right\}_{\tau<t_{0}}$ is uniformly bounded and hence equicontinuous on every compact set in $\Omega^{\left(t_{0}\right)}$. Then there exist a decreasing sequence $\left\{\tau_{k}\right\}_{k=1}^{\infty}$ tending to $-\infty$ and a solution $u$ of $L u=0$ on $\Omega$ such that

$$
\lim _{k \rightarrow \infty} u_{\tau_{k}}=u \quad \text { (compact uniformly). }
$$

Using Lemma 5 for $u_{\tau_{k}}$ and letting $k$ tend to the infinity, we see that $u$ vanishes continuously on $\partial \Omega$, so that $u \in H_{0}(\Omega, L)$. This completes the proof.

\section{§4. The uniqueness of positive solutions}

Let $D$ be a bounded Lipschitz domain in $R^{n}$ and $\varphi$ a strictly positive $1 / 2$-Hölder continuous function on $R$. 
Remark 2. $\Omega(D, \varphi)$ is a $(1,1 / 2)$-Lipschitz domain with Lipschitz constant $\max (c, m(1+c) d(0, \partial D))$, where $c$ is the Lipschitz constant of $D, m$ is the $1 / 2$-Hölder constant and $d(0, \partial D)$ is the distance from 0 to $\partial D$.

The following lemma is a kind of boundary Harnack principle.

Lemma 6. For a bounded Lipschitz domain $D$ in $R^{n}$ and a 1/2-Hölder continuous function $\varphi>0$ on $R$, we put $\Omega=\Omega(D, \varphi)$. Let $\tau_{0}>0, \tau \in R$ and $\Delta$ be a non-empty subdomain of $D$ with $\bar{\Delta} \subset D$. Then there exists a constant $C>0$ independent of $\tau$ such that

$$
\sup _{\varphi(\tau) D \times\{\tau\}} u \leq C \inf _{\varphi(\tau) \Delta \times\{\tau\}} u
$$

for every solution $u \geq 0$ of $L u=0$ on $\Omega^{\left(\tau-\tau_{0} \varphi(\tau)^{2}\right)}$ which vanishes continuously on $\partial \Omega \cap R^{n} \times\left[\tau-\tau_{0} \varphi(\tau)^{2}, \infty\right)$.

Proof. Let $x_{0} \in \Delta$ be fixed. Put $t_{0}=\left(\tau_{0}^{-1 / 2}+m\right)^{-2}$, where $m$ is the $1 / 2$-Hölder constant of $\varphi$. Then there exists $0<T \leq \tau_{0} \varphi(\tau)^{2}$ such that

$$
\frac{T}{\varphi(\tau-T)^{2}}=t_{0} .
$$

Applying Lemma 4 to $\tau_{0}=t_{0} / 2$ and using the parabolic dilation $\tau_{\varphi(\tau-T)}$, we have for any solution $u \geq 0$ of $L u=0$ on $\Omega^{\left(\tau-\tau_{0} \varphi(\tau)^{2}\right)}$ which vanishes continuously on $\partial \Omega \cap R^{n} \times\left[\tau-\tau_{0} \varphi(\tau)^{2}, \infty\right)$,

$$
\sup _{x \in \varphi(\tau) D} u(x, \tau) \leq C_{1} u\left(\varphi\left(\tau-\frac{T}{2}\right) x_{0}, \tau-\frac{T}{2}\right) \leq C_{1} C_{2} \inf _{x \in \varphi(\tau) \Delta} u(x, \tau),
$$

which shows Lemma 6.

Let $L^{*}$ be the adjoint operator of $L \in \mathscr{L}(M)$. Then for any solution $u$ of $L^{*} u=0, v(x, t)=u(x,-t)$ is a solution of $\tilde{L} v=0$ for some $\tilde{L} \in \mathscr{L}(M)$, so that the analogous assertions to Lemma 6 hold. This yields Lemma 7 , which plays an important role to show the uniqueness.

Lemma 7. For a bounded Lipschitz domain $D$ in $R^{n}$ and a 1/2-Hölder continuous function $\varphi>0$ on $R$, we put $\Omega=\Omega(D, \varphi)$. Let $\tau_{0}>0, \tau \in R$ and $\Delta$ be a non-empty subdomain of $D$ with $\bar{\Delta} \subset D$. Then there exists a constant $C>0$ independent of $\tau$ such that 


$$
\omega_{\Omega^{(\tau)}}^{(x, t)}(\varphi(\tau) D \times\{\tau\}) \leq C \omega_{\Omega^{(\tau)}}^{(x, t)}(\varphi(\tau) \Delta \times\{\tau\})
$$

for every $(x, t) \in \Omega^{\left(\tau+\tau_{0} \varphi(\tau)^{2}\right)}$.

Proof. Let $G(x, t ; y, s)$ be the Green function of $L$ with respect to $\Omega(D, \varphi)$. Then for $(x, t) \in \Omega(D, \varphi)$,

$$
\omega_{\Omega^{(\tau)}}^{(x, t)}=G(x, t ; y, \tau) d y \text { on } \varphi(\tau) D \times\{\tau\},
$$

where $d y$ denotes the $n$-dimensional Lebesgue measure. For $(x, t) \in \Omega^{\left(\tau+\tau_{0} \varphi(\tau)^{2}\right)}$, $G(x, t ; \cdot, \cdot)$ is a solution of the adjoint operator $L^{*}$ of $L$ on $\Omega \cap R^{n} \times$ $(-\infty, t)$. Applying Lemma 6 to $L^{*}$, we obtain

$$
\sup _{y \in \varphi(\tau) D} G(x, t ; y, \tau) \leq C \inf _{y \in \varphi(\tau) \Delta} G(x, t ; y, \tau),
$$

which shows our lemma.

We shall show our main theorem, which implies the preceding assertion in the paragraph 1 .

THEOREM 2. Let $D$ be a bounded Lipschitz domain in $R^{n}$ and $\varphi>0$ a locally $1 / 2$-Hölder continuous function on $(-\infty, a)$ with $a \in(-\infty, \infty]$. Suppose that there exist $m>0, \tau_{0}>0$ and a sequence $\left\{t_{k}\right\}_{k=1}^{\infty}$ tending to $-\infty$ as $k \rightarrow \infty$ such that

$$
\liminf _{k \rightarrow \infty}\left|t_{k}\right|^{-1 / 2} \varphi\left(t_{k}\right)<\infty
$$

and that for every $k=1,2, \ldots$,

$$
|\varphi(t)-\varphi(s)|<m|t-s|^{1 / 2}
$$

for $t, s \in\left[t_{k}, t_{k}+\tau_{0} \varphi\left(t_{k}\right)^{2}\right]$. Then there exists $u \neq 0$ such that

$$
H_{0}(\Omega(D, \varphi), L)=\{c u ; c \geq 0\} .
$$

Proof. By Proposition 1 and Remark 2, $H_{0}(\Omega(D, \varphi), L) \neq\{0\}$. Hence it suffices to show that there exist $C>0$ and $h \in H_{0}(\Omega(D, \varphi), L)$ with $h\left(Y_{0}\right)=1$ for fixed $Y_{0} \in \Omega(D, \varphi)$ such that $u \geq C h$ for every $u \in H_{0}(\Omega(D, \varphi), L)$ with $u\left(Y_{0}\right)$ (see [3], p.253).

Let $u \in H_{0}(\Omega(D, \varphi), L)$ with $u\left(Y_{0}\right)=1$ and put $\Omega=\Omega(D, \varphi)$. Taking a subsequence of $\left\{t_{k}\right\}_{k=1}^{\infty}$ and replacing $\tau_{0}$ by smaller one if necessary, we may 
assume that

$$
t_{k}+\tau_{0} \varphi\left(t_{k}\right)^{2}<\frac{t_{k}}{2}
$$

for every positive integer $k$. Put

$$
T_{k}=t_{k}+\frac{\tau_{0} \varphi\left(t_{k}\right)^{2}}{2}
$$

Let $\Delta$ be a non-empty subdomain of $D$ and take $x_{0} \in \Delta$. Then by Lemmas 6 and 7 , we have for every positive integer $k$ and every $(x, t) \in \boldsymbol{\Omega}^{\left(t_{k}+\tau_{0} \varphi\left(t_{k}\right)^{2}\right)}$

$$
\begin{aligned}
u(x, t) & =\int_{\varphi\left(T_{k}\right) D \times\left\{T_{k}\right\}} u\left(y, T_{k}\right) d \omega_{\Omega^{\left(T_{k}\right)}}^{(x, t)}(y) \\
& \geq \int_{\varphi\left(T_{k}\right) \Delta \times\left\{T_{k}\right\}} u\left(y, T_{k}\right) d \omega_{\left.\Omega^{\left(T_{k}\right.}\right)}^{(x, t)}(y) \\
& \geq\left(\inf _{\varphi\left(T_{k}\right) \Delta \times\left\{T_{k}\right\}} u\right) \omega_{\Omega^{\left(T_{k}\right)}}^{(x, t)}\left(\varphi\left(T_{k}\right) \Delta \times\left\{T_{k}\right\}\right) \\
& \geq C_{1}^{-1} u\left(\varphi\left(T_{k}\right) x_{0}, T_{k}\right) \omega_{\Omega^{\prime}\left(T_{k}\right)}^{(x, t)}\left(\varphi\left(T_{k}\right) D \times\left\{T_{k}\right\}\right),
\end{aligned}
$$

where $C_{1}>0$ is a constant independent of $k, u$ and $(x, t)$. On the other hand, by Lemma 5 , there exists a constant $C_{2}>0$ such that

$$
1=u\left(Y_{0}\right) \leq C_{2} u\left(\varphi\left(T_{k}\right) x_{0}, T_{k}\right) \omega_{\Omega^{\left(T_{k}\right)}}^{Y_{0}}\left(\varphi\left(T_{k}\right) D \times\left\{T_{k}\right\}\right),
$$

so that

$$
u \geq C_{1}^{-1} C_{2}^{-1} h_{k} \quad \text { on } \Omega^{\left(t_{k}+\tau_{0} \varphi\left(t_{k}\right)^{2}\right)},
$$

where

$$
h_{k}(x, t)=\frac{\omega_{\Omega^{\left(T_{k}\right)}}^{(x, t)}\left(\varphi\left(T_{k}\right) D \times\left\{T_{k}\right\}\right)}{\omega_{\Omega^{\left(T_{k}\right)}}^{Y_{0}}\left(\varphi\left(T_{k}\right) D \times\left\{T_{k}\right\}\right)} .
$$

Similarly to Proposition 2, we can take a subsequence of $\left\{h_{n}\right\}_{n=1}^{\infty}$ which converges a certain $h \in H_{0}(\Omega, L)$ with $h\left(Y_{0}\right)=1$, which shows

$$
u \geq C_{1}^{-1} C_{2}^{-1} h \text { on } \Omega \text {. }
$$

This completes the proof.

Remark 3. The assumptions (7), (8) in Theorem 2 can be replaced by

$$
|\varphi(t)-\varphi(s)|<m|t-s|^{1 / 2}
$$


for $t, s \in\left[t_{k}-\tau_{0} \varphi\left(t_{k}\right)^{2}, t_{k}+\tau_{0} \varphi\left(t_{k}\right)^{2}\right]$.

Applying Theorem 1 to $\varphi_{\alpha}(t)=(-t)^{\alpha}(t<0)$, we have

Corollary. Let $-\infty<\alpha \leq 1 / 2$. For a bounded Lipschitz domain $D$ in $R^{n}$, put

$$
\Omega_{\alpha}=\left\{(x, t) ; t<0,(-t)^{-\alpha} x \in D\right\} .
$$

Then every non-zero elements in $H_{0}\left(\Omega_{\alpha}, L\right)$ are mutually proportional.

Example. Let $D$ be a bounded Lipschitz domain in $R^{n}$ and put $\Omega=D \times R$. Then

$$
H_{0}\left(\Omega, \frac{\partial}{\partial t}-\Delta\right)=\left\{c e^{-\lambda t} f(x) ; c \geq 0\right\}
$$

where $\lambda$ is the first eigenvalue of $-\Delta$ (Laplacian) and $f$ is the eigenfunction.

\section{REFERENCES}

[1] D.G. Aronson, Non-negative solutions of linear parabolic equations, Ann. Scuola Norm. Sup. Pisa, 22 (1968), 607-694.

[2] E.B. Fabes, N. Garofalo and S. Salsa, A backward Harnack inequality and Fatou theorem for nonnnegative solutions of parabolic equations, Illinois J. Math., 30 (1986), 536-565.

[3] J.T. Kemper, Temperatures in several variables: kernel functions, representations, and parabolic boundary values, Trans. Amer. Math. Soc., 167 (1972), 243-262.

[4] J. Moser, A Harnack inequality for parabolic differential equations, Comm. Pure Appl. Math., 17 (1964), 101-134.

Department of Mathematics

Osaka City University

Sugimoto, Sumiyoshi

Osaka 558, Japan 